\title{
Impaired contrast sensitivity in diabetic patients with and without retinopathy: a new technique for rapid assessment
}

\author{
S DELLA SALA, ${ }^{1}$ G BERTONI, ${ }^{2}$ L SOMAZZI, ${ }^{2}$ F STUBBE, ${ }^{3}$ AND A J WILKINS ${ }^{4}$ \\ From the '1st Department of Neurology, University of Milan, $S$ Paolo Hospital, Via di Rudini, 8, 20142 Milan, \\ Italy; the ${ }^{2}$ Department of Ophthalmology, University of Milan, L Sacco Hospital, Via G B Grassi 74, 20100, \\ Milan, Italy; ${ }^{3}$ Instituut voor Epilepsiebestrijding, Heemstede, The Netherlands; and the ${ }^{4} M R C$ Applied \\ Psychology Unit, 15 Chaucer Road, Cambridge CB2 2EF, England
}

SUMmaRY A simple technique is described for assessing the sensitivity of the human visual system to gratings at threshold contrast. The technique has advantages for clinical use in that it is (1) inexpensive, (2) quick to administer, (3) portable, and (4) relatively free from bias. Forty-two diabetic patients and 84 normal controls have been tested. Fifteen diabetic patients $(6 / 20$ with retinopathy and $9 / 22$ without) had test scores more than two standard deviations below the norm for age-matched controls.

The threshold contrast at which a pattern of stripes is just visible is a measure of visual function that has recently received great attention. It differs from conventional acuity in several respects ${ }^{1-3}$ and can pick up deficits not detected by more conventional measures in lens $^{4-5}$ and retinal ${ }^{6-10}$ pathology, glaucoma, ${ }^{11-15}$ retrobulbar neuritis, ${ }^{16-18}$ and other disorders. ${ }^{319-21}$ The contrast threshold is best measured by electronically varying the contrast of a pattern on the surface of a cathode ray tube, ${ }^{22-24}$ but such a system is not convenient for widespread clinical use. Arden ${ }^{25}$ has therefore devised a test consisting of gratings printed on cards. The contrast of the gratings decreases along the length of the stripes. The subject covers the grating with a card, exposing the lowest contrast first, until he reports that he can see the stripes. The test has its clinical utility 11.525 but is expensive to produce, costing about $\$ 200$ (£135) to purchase. This method of measuring contrast is open to the objection that strategic differences in criterion may have a considerable and indeterminate influence. By cutting the Arden plates into discs Vaegan and Halliday ${ }^{26}$ devised a printed contrast sensitivity test with four-alternative forced choice format, in which subjects were required to report the orientation of the stripes.

Correspondence to Dr A J Wilkins.
The aim of this article is to describe a simple twoalternative forced choice test which is both quick to administer and cheap to reproduce. It differs from the other tests in seeking to obtain a rapid but reliable estimate of the detectability of gratings with just one spatial frequency, namely 4 cycles/degree, the frequency at which the sensitivity of the visual system is near its maximum..$^{23}$ The orientation of the gratings is usually horizontal but can be varied if required.

Arden, ${ }^{27}$ Ghafour et al.,${ }^{6}$ Hirsch and Puklin, ${ }^{28}$ and Hyvärinen et al. ${ }^{7}$ have reported that contrast sensitivity is reduced in diabetic patients. Our test was developed to investigate diabetic patients with normal Snellen acuity. All the patients received a complete ophthalmological examination on the basis of which they were divided into two groups: those with and those without retinopathy.

\section{Details of the test}

The test comprises a series of 10 pairs of square plates. The pairs are presented with one plate above the other, the grating in the top or the bottom plate at random. The observer is forced to choose whether the top or the bottom plate contains the grating, guessing if necessary. The first pair of the series has a grating with high contrast which serves as an example, and the gratings in subsequent plates 


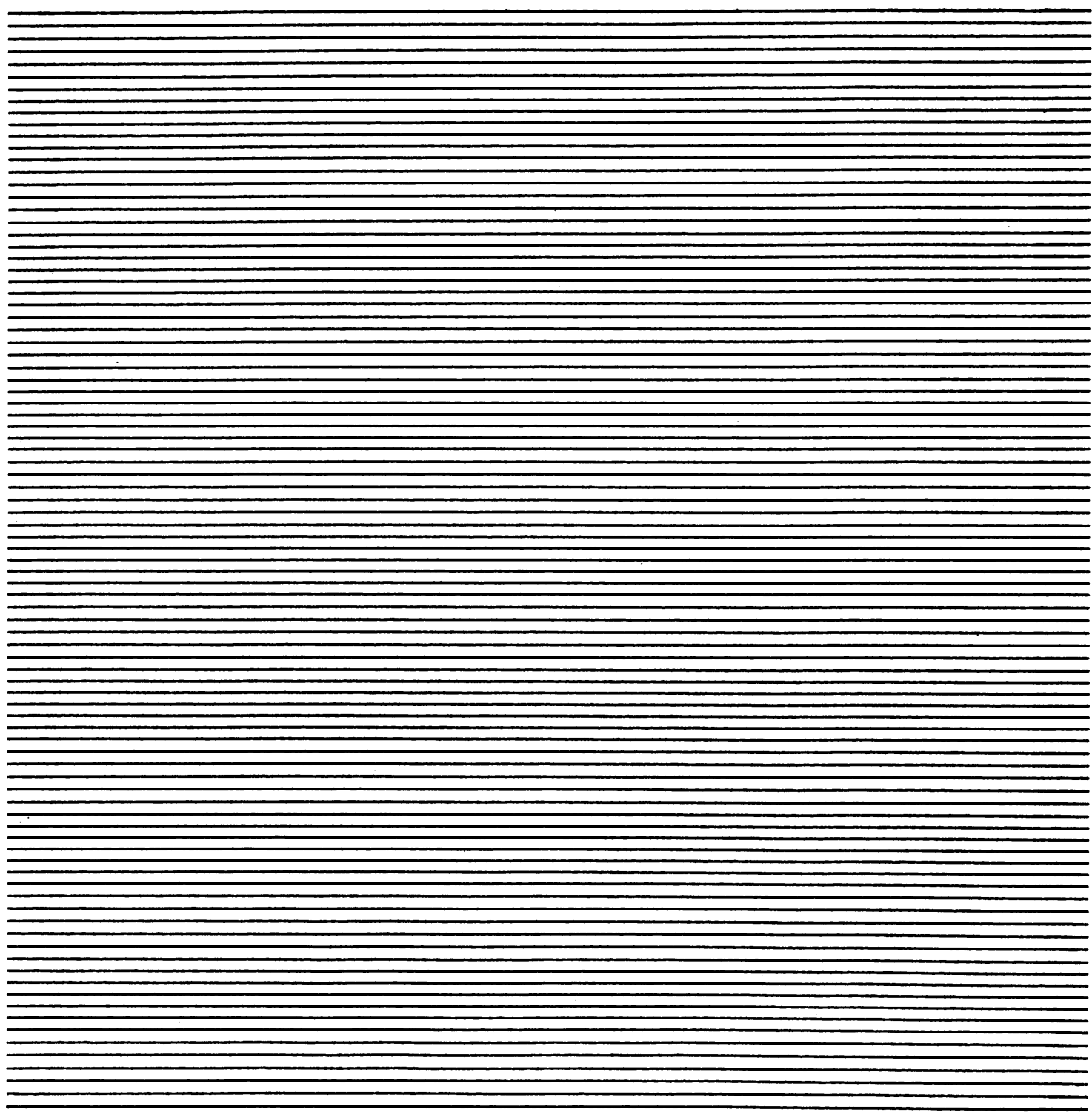

Fig. 1 An apparent grating with square-wave luminance profile resulting from variations in the spacing of the lines, suitable for viewing at $6 \mathrm{~m}$.

decrease in contrast. The plates are designed to be viewed at $6 \mathrm{~m}$, at which distance they subtend $2^{\circ}$ by $2^{\circ}$ and have a spatial frequency of 4 cycles/degree. (In the present study the plates were reduced in size by $33 \%$ by means of electrostatic photo-reduction so that they could be viewed at $5 \mathrm{~m}$, the distance at which visual acuity is usually measured in Italy. They subtended $1.5^{\circ} \times 1.5^{\circ}$.)

The test plates are printed from computer- generated plots of parallel lines that vary in their separation and thus their density, as shown in Fig. 1. They have a luminance profile that has been represented schematically in Fig. 2.

At the distance from which the plates are viewed the individual lines can no longer be seen. The variations in line density are, however, visible. These variations in density themselves comprise stripes (apparent gratings with a square-wave luminance 

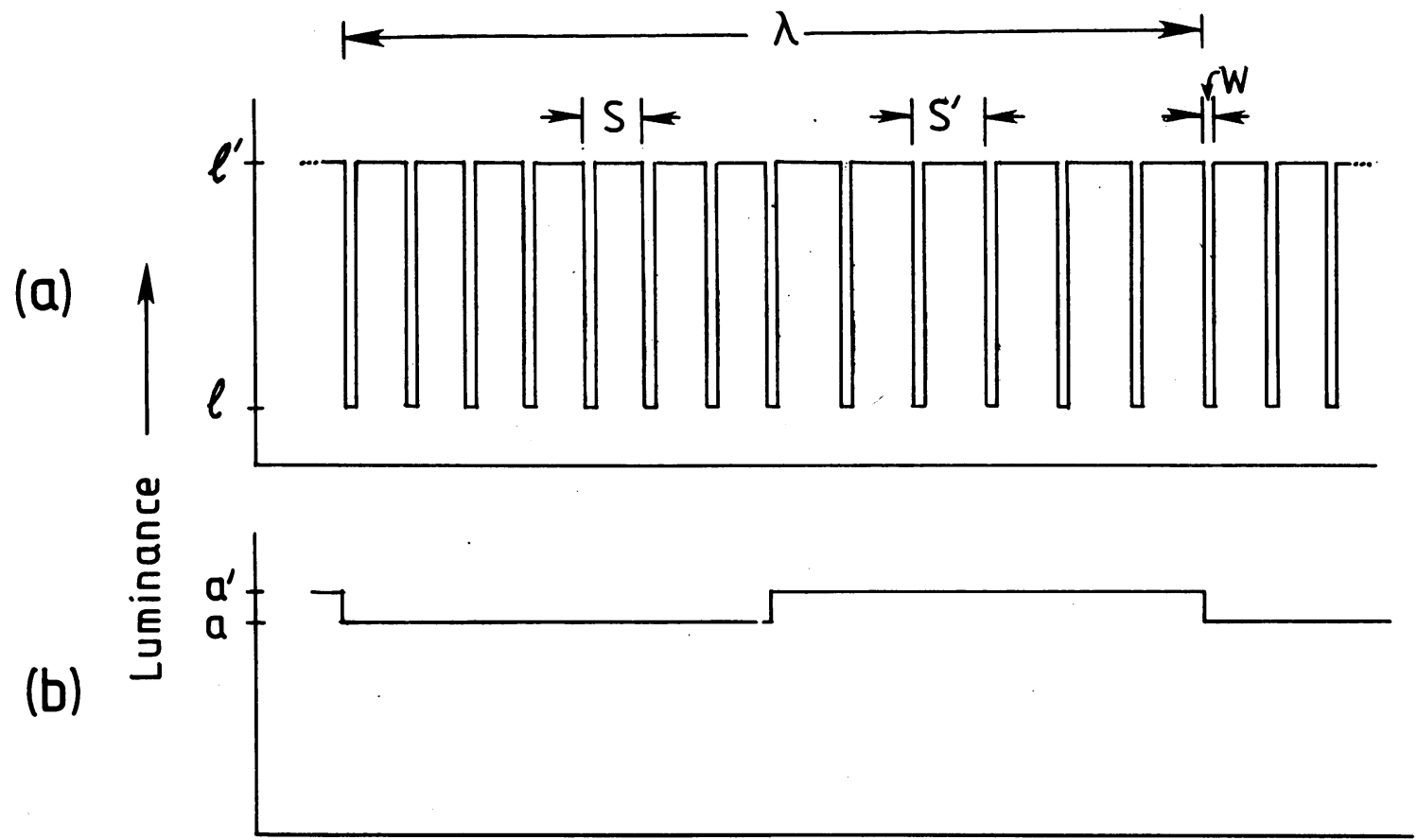

\section{Space $\longrightarrow$}

Fig. 2a Luminance profile of the grating shown in Fig. 1. The ink in which it is printed has a luminace of l, and the paper a luminance of $l^{\prime}$. The spacing of the lines (width $w$ ) alternates between $s$ and $s^{\prime}$ every $\lambda$ units of length so as to change the line density with a square-wave luminance profile shown in Fig. $2 b$. When viewed from a distance at which the component lines cannot be resolved, the grating has an apparent contrast of $\left(a^{\prime}-a\right) /\left(a^{\prime}+a\right)$ and a spatial frequency of $l / \lambda$.

profile and a period of $36 \mathrm{~mm}-$ i.e., a spatial frequency of 28 cycles $/ \mathrm{m}$. The Michelson contrast of the apparent grating is given by the expression

$$
\left(a^{\prime}-a\right) /\left(a^{\prime}+a\right)
$$

where $a$ and $a^{\prime}$ are the mean reflectances of the closely spaced and widely spaced lines respectively. The mean reflectance is determined by the reflectance of the paper, the reflectance of the ink, and the width and spacing of the lines. It can be shown that the above expression reduces to

$$
\frac{c w\left(s^{\prime}-s\right)}{(1+c) s s^{\prime}-c w\left(s+s^{\prime}\right)}
$$

where $c$ is the contrast of the ink on the paper, $w$ is the width of the lines, and s' and $s$ are the maximum and minimum separation of the lines respectively (see Fig. 2). From the above expression it can be shown that the contrast of the apparent grating will change little with changes in the contrast of the ink on the page or the width of the individual lines, factors that might be expected to vary with printing impression. For values of the parameters within the range used, changes of $10 \%$ in the width of the lines or the contrast of the ink on the page change the contrast of the apparent grating by a similar amount. Contrast sensitivity in normal persons may vary by a factor of 2 or more, for example, between $0.5 \%$ and $1 \%$. If an apparent grating is made with a contrast of $0.5 \%$, and the line width or contrast of the ink alters by, say, $20 \%$ (which is more than would occur in practice), then the contrast of the apparent grating would be expected to vary between $0 \cdot 4 \%$ and $0 \cdot 6 \%$, depending on the direction of variation in these parameters and the extent to which they covaried. The changes in apparent contrast from one printing impression to another are therefore likely to have little effect on the percentile scores obtained by the test and thus on its ability to discriminate normal from abnormal vision. The contrast of the apparent grating is critically determined by the separation of successive lines, and this should vary little with printing impression.

As mentioned above, the test plates were reduced for use at $5 \mathrm{~m}$. The line separations are shown after 
Table 1 Reduced version of low contrast plates for use at $5 \mathrm{~m}$

\begin{tabular}{llll}
\hline $\begin{array}{l}\text { Plate } \\
\text { pair }\end{array}$ & \multicolumn{2}{l}{\begin{tabular}{l} 
Separation $(\mathrm{mm})^{*}$ \\
Mominal \\
\cline { 2 - 3 }
\end{tabular}} & $\begin{array}{l}\text { Momimum } \\
\text { contrast }(\%)\end{array}$ \\
\hline 1 & 1.57 & 1.33 & \\
2 & 1.51 & 1.33 & 2.07 \\
3 & 1.45 & 1.33 & 1.62 \\
4 & 1.43 & 1.33 & 1.13 \\
5 & 1.40 & 1.33 & 0.96 \\
6 & 1.39 & 1.33 & 0.69 \\
7 & 1.37 & 1.33 & 0.59 \\
8 & 1.36 & 1.33 & 0.40 \\
9 & 1.35 & 1.33 & 0.30 \\
Blank & 1.33 & 1.33 & 0.20 \\
\hline
\end{tabular}

*Values calculated on the basis of the photoreduction.

reduction in Table 1 , together with a nominal value for the contrast of the apparent grating based on a value of 0.7 for the contrast of the ink on the paper and a value of $0.35 \mathrm{~mm}$ for the width of the lines. The separation of lines was chosen so that the apparent contrast of successive gratings decreased with intervals on a logarithmic scale that were as evenly spaced as the technical constraints would allow. (In the light of experience with the test a revised version has now been produced for use at $6 \mathrm{~m}$, and the details of this test are shown in Table 2.)

The contrast of the apparent grating was measured for the photocopies of the first two test plates by means of a bifurcated optic fibre to project light to, and collect light from, a rectangular portion of the plate measuring $10 \times 70 \mathrm{~mm}$, orientated with the long axis parallel to the lines. The contrasts of the first two plates were within 0.2 of the values tabulated. The nominal values of contrast may therefore be taken to be reasonable estimates of the actual values.

\section{Subjects and methods}

Forty-two diabetic patients, 22 male and 20 female, aged 12-75 and 84 normal volunteers, 52 male and 32 female, aged 14-68 took part in the study. All subjects (patients and volunteers) were selected as having a Snellen acuity of 1.0 or more $(5 / 5$ as it is usually measured in Italy), after correction where necessary (no subject was amblyopic). The diabetic patients were chosen from those patients who regularly attended the eye clinic at L Sacco Hospital, University of Milan. Hospital staff, medical students, and patients' relatives volunteered to act as unpaid (age matched) control subjects.

All subjects underwent a full ophthalmological investigation which included Snellen acuity, slit-lamp examination, tonometry, and direct and indirect ophthalmoscopy. The patients were also examined
Table 2 Revised version of low contrast plates for use at $6 \mathrm{~m}$

\begin{tabular}{llll}
\hline $\begin{array}{l}\text { Plate } \\
\text { pair }\end{array}$ & \multicolumn{2}{l}{$\begin{array}{l}\text { Separation }(\mathrm{mm}) \\
\text { Maximum }\end{array}$} & $\begin{array}{l}\text { Nominal } \\
\text { contrast (\%) }\end{array}$ \\
\cline { 2 - 4 } & Minum & \\
\hline 1 & 2.22 & 1.38 & 4.76 \\
2 & 2.06 & 1.54 & 2.82 \\
3 & 1.96 & 1.64 & 1.71 \\
4 & 1.90 & 1.70 & 1.06 \\
5 & 1.86 & 1.74 & 0.64 \\
6 & 1.84 & 1.76 & 0.42 \\
7 & 1.82 & 1.78 & 0.21 \\
Blank & 1.80 & 1.80 & 0.00 \\
\hline
\end{tabular}

by Goldmann lens biomicroscopy and fluorescein angiography. In none of the subjects who were admitted to the study could we detect any ocular alteration such as glaucoma, cataract, or minimal opacities, although 34 normals and 24 patients needed optical correction.

Twenty-two patients (11 male and 11 female aged 17-72, mean 46) had no detectable retinopathy. Fluorescein angiography showed no evidence of microaneurysms, haemorrhages, soft or hard exudates, areas of capillary non-perfusion, or leakage of vessels at the posterior pole or in the periphery of either eye. The length of the patients' illness ranged from 1 to 22 years (mean 7 years). Eight were affected by type I diabetes mellitus and 14 by type II. ${ }^{29}$

Twenty patients (11 male and 9 female, aged 16-75, mean 42) had retinopathy. Nineteen had a background retinopathy (microaneurysms, scattered haemorrhages, and a few hard exudates at the posterior pole) and one, a male aged 48 , had proliferative retinopathy with an initial peripheral neovascularisation. In all but one, a 17-year-old female, the retinopathy was bilateral. In three there was an initial macular oedema with retention of good visual acuity. Two other patients presented areas of capillary non-perfusion at the periphery of the fundus. The contrast test was administered prior to argon laser photocoagulation when this was required. The length of the patients' illness ranged from 3 to 31 years (mean 15 years). Twelve patients were affected by type I and eight by type II diabetes.

The test plates were mounted on the pages of a spiral bound book. The book was fixed on a wooden stand at a viewing distance of $5 \mathrm{~m}$ and illuminated by a filament lamp (60 watts at a distance of $1.5 \mathrm{~m}$ ) so that the mean luminance was about $50 \mathrm{~cd} / \mathrm{m}^{2}$. Care was taken to ensure that the position of the lamp, plates, and eye of the observer did not vary from one test to the next. The series of plates was presented three times. Eyes were tested in random order. 


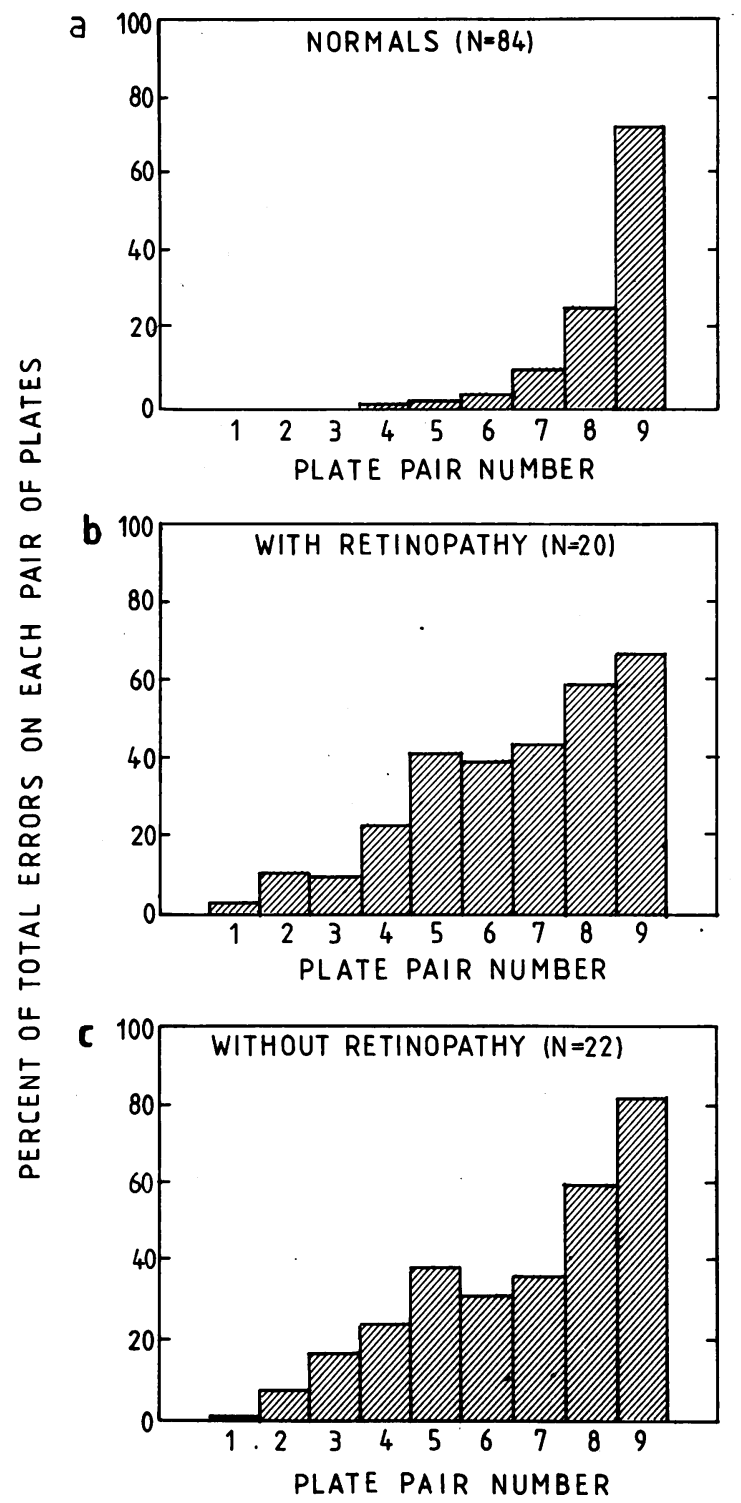

Fig. 3 Histogram of the number of errors made on stimulus pairs 1-9, for (a) normal control subjects, (b) diabetic patients with and (c) without retinopathy.

The data for control subjects and patients were collected over the same period of time (about one year), and so any degradation of the plates that may have occurred could not have accounted for the differences between groups.

\section{Results}

In Fig. 3 the errors made by each group of subjects are shown separately for the nine pairs of test plates. From these histograms it is evident that on the pair of plates with the lowest contrast stimulus (pair number 9), subjects were not responding to the grating but to some aspect of the blank stimulus that resembled a grating (see Details of test). Were subjects responding at random, that is, appropriately for a grating they could not see; an error rate of about $50 \%$ would have been expected. Both normal subjects and patient groups responded with an overall error rate of about $75 \%$, a rate significantly above chance. The responses to this pair of stimuli have been included in the analyses that follow because when they were removed the findings were not appreciably changed.

An initial set of analyses was performed on the total number of errors made by the two eyes on the three presentations of the test. Preliminary analyses of the data for normal subjects failed to reveal any sex differences but indicated an increase in errors with age, maximal over the age of 50 . On the basis of these analyses the normal subjects were divided into three groups: 40 young (14-29, mean 22): 30 middle-aged (31-48, mean 40$) ; 14$ older (50-68, mean 59). The total error scores for both eyes averaged: $5 \cdot 2$ (SD $2 \cdot 5), 7 \cdot 3$ (SD 5.2), and 11.0 (SD 7.6) for the three age groups respectively. On the basis that the percentage correct responses to plates 7 and 8 were respectively slightly more and slightly less than $75 \%$, the mean contrast sensitivity (reciprocal of contrast threshold) for the group was between 250 and 330 . For the patients the total error scores were converted to $\mathrm{z}$ scores by comparison with the appropriate age matched control group. Six of the 20 patients with retinopathy and nine of the 22 without had total error scores more than 2 standard deviations above the mean for the normals (see Table 3 ). There were no differences between the error scores for patients with type I and type II diabetes or those with and without retinopathy. The lower proportion of older patients with poor $\mathrm{z}$ scores is presumably because of the

Table 3 Number of patients in each age group (size n) with and without retinopathy, whose error scores were more than 2 standard deviations above the mean for age matched controls. The scores were calculated on the basis of the total errors for both eyes on all trials, total errors for the worst eye on all trials, and errors for both eyes on the first trial only

\begin{tabular}{|c|c|c|c|c|c|c|c|c|}
\hline \multirow[t]{2}{*}{ Age range } & \multicolumn{3}{|c|}{ With retinopathy } & \multirow[t]{2}{*}{$n$} & \multicolumn{3}{|c|}{ Without retinopathy } & \multirow[t]{2}{*}{$n$} \\
\hline & Total & Worst & First & & Total & Worst & First & \\
\hline $10-29$ & 1 & 1 & 1 & 2 & 5 & 5 & 5 & 7 \\
\hline $30-49$ & 4 & 4 & 4 & 6 & 3 & 4 & 2 & 5 \\
\hline $50+$ & 1 & 1 & 2 & 12 & 1 & 1 & 1 & 10 \\
\hline Total & 6 & 6 & 7 & 20 & 9 & 10 & 8 & 22 \\
\hline
\end{tabular}


greater variability between normal individuals in the older age group.

Similar analyses were also performed using the scores for the eye with the highest error score, and these produced closely comparable results (see Table 3).

In both of these analyses the correlation between the number of errors and the length of the disease was positive (Spearman rank correlation between 0.2 and $0 \cdot 3$ ) but did not approach significance, regardless of the presence or absence of retinopathy.

When the error scores for the two eyes on the first presentation of the test were considered separately, they were highly correlated with the total errors on all presentations (normals: $r_{s} 0 \cdot 85$; patients: $r_{s} 0 \cdot 93$ ). As can be seen from a comparison of the data in Table 3 , a single presentation of the test to each eye (taking about one minute per eye) would have been sufficient to have detected all but one of the nine patients without retinopathy who had an impaired contrast sensitivity.

\section{Discussion}

All the patients had a Snellen acuity of $5 / 5$ or better, corrected if necessary. Nevertheless, nine of the 22 without retinopathy and six of the 20 with retinopathy had contrast test scores more than 2 standard deviations below the norm. No comparable figures are available from previous studies, although Ghafour $e t$ $a l .{ }^{6}$ report a significant difference between normals and diabetic patients without retinopathy as well as those with. In neither the present study nor those previous to it was impairment in contrast sensitivity ${ }^{628}$ or other visual function ${ }^{311}$ related to observable retinal pathology (except when the pathology was gross ${ }^{7}$ ), possibly because the retinopathy is often very localised. The test we have described measures visual function within the central $1.5^{\circ}$ of the visual field. If impaired function precedes clinically evident anatomical changes, it is likely that those patients whose contrast sensitivity was impaired may later show background retinopathy.

Despite its simplicity and the speed with which it could be administered our test was sensitive enough to show the expected decline in sensitivity with age a $^{31-35}$ and to reveal an impaired sensitivity in certain diabetic patients. ${ }^{6728}$ There is a high test-retest reliability, perhaps because the forced choice procedure reduced variation due to changes in the subjects' strategy and criterion. ${ }^{26}{ }^{32} 36$ The test is inexpensive, portable, and easy to score, and it can examine contrast sensitivity in any orientational meridian. Although it can measure contrast threshold at only one spatial frequency, the frequency chosen is that at which the visual system is most sensitive ${ }^{23}$ and which has been shown to discriminate diabetic patients from normal subjects. ${ }^{6}$ The test is designed to be administered only to patients who have normal Snellen acuity, but this restriction is less of a disadvantage than it might seem, given that the test is useful in detecting deficits that are not measurable by other clinical methods. ${ }^{37}$

The authors thank J G Robson and I Nimmo-Smith for their advice, $P$ Hall, G Plant and their colleagues in the EEG department at Addenbrookes Hospital, Cambridge, for trying out a preliminary version of the test, $M$ Barrett for assistance with the contrast measurements, and C D Binnie for the use of computing resources.

NoTE. A new version of the test, using letters, is currently in preparation in collaboration with Dr J G Robson. Copies of this test, and of the test described here, can be obtained for a nominal charge by writing to the last-named author.

\section{References}

1 Bodis-Wollner I. Detection of visual defects using the contrast sensitivity function. Int Ophthalmol Clin 1980; 20: 135-53.

2 Bodis-Wollner $I$. Visual acuity and contrast sensitivity in patients with cerebral lesions. Science 1972; 178: 769-71.

3 Bodis-Wollner I, Diamond SP. The measurement of spatial contrast sensitivity in cases of blurred vision associated with cerebral lesions. Brain 1976; 99: 695-710.

4 Paulsson LE, Stostrand J. Contrast sensitivity in the presence of a glare light: theoretical concepts and preliminary clinical studies. Invest Ophthalmol Visual Sci 1980; 19: 401-6.

5 Hesse RF, Woo G. Vision through cataracts. Invest Ophthalmol Visual Sci 1978; 17: 428-36.

6 Ghafour M, Foulds WS, Allan D, McClure F. Contrast sensitivity in diabetic subjects with and without retinopathy. BrJ Ophthalmol 1982; 66: 492-5.

7 Hyvärinen L, Laurinen P, Rovamo J. Contrast sensitivity in evaluation of visual impairment due to diabetes. Acta Ophthalmol 1983; 61: 94-101.

8 Wolkstein M, Atkin A, Bodis-Wollner I. Contrast sensitivity in retinal disease. Ophthalmology 1980; 87: 1140-9.

9 Shalka HW. Comparison of Snellen acuity, VER acuity and Arden grating scores in macular and optic nerve diseases. $\mathrm{Br} J$ Ophthalmol 1980; 64: 24-9.

10 Sjostrand T. Contrast sensitivity in macular disease using a smallfield and a large-field TV system. Acta Ophthalmol (Kbh) 1979; 57: $832-46$.

11 Arden GB, Jacobson JJ. A simple grating test for contrast sensitivity; preliminary results indicate value in screening for glaucoma. Invest Ophthalmol Visual Sci 1978; 17: 23-32.

12 Atkin A, Wolkstein M, Bodis-Wollner I, Anders M, Kels B, Podos S. Interocular comparison of contrast sensitivitics in glaucoma patients and suspects. Br J Ophthalmol 1980; 64: 858-62.

13 Cooper RL, Constable IJ, Terrell A. Mass screening for glaucoma and other eye disease using the Arden grating test. Aust J Ophthalmol 1980; 8: 131-7.

14 Editorial. Contrast sensitivity revisited. Br J Ophthalmol 1981; 65: 513-4.

15 Singh H, Cooper RL, Alder VA, Crawford GJ, Terrell A, Constable IJ. The Arden grating acuity: effect of age and optical factors in the normal patient with prediction of the false negative in screcning for glaucoma. Br J Ophthalmol 1981; 65: 518-24.

16 Regan D, Silver R, Murray TG. Visual acuity and contrast sensitivity in multiple sclerosis: hidden visual loss. Brain 1977; 100: $563-79$.

17 Zanen A. Diminution de la sensibilité au contrast spatial èt stéréoillusion de Pulrich chez des patients atteints de sclérose en plaques. Bull Soc Belge Ophtalmol 1982; 199-200: 95-115. 
18 Zimmern RL, Campbell FW, Wilkinson IM. Subtle disturbance of vision after optic neuritis elicited by studying contrast sensitivity. J Neurol Neurosurg Psychiatry 1972; 42: 407-12.

19 Hcss RF, Carney LG. Vision through an abnormal cornea: a pilot study of the relationship between visual loss from corneal dislocation, corneal edema, keratoconus and some allied corneal pathology. Invest Ophthalmol Visual Sci 1979; 18: 476-83.

20 Bender MD, Bodis-Wollner I. Visual dysfunction in optic tract lesions. Ann Neurol 1978; 3: 187-93.

21 Marron JA, Bailey IL. Visual factors and orientation-mobility performance. Am J Optom Physiol Opt 1982; 59: 413-26.

22 Campbell FKW, Maffel I. Contrast and spatial frequency. Sci Am 1974; 231: 106-14.

23 Campbell FKW, Robson JG. Application of Fourier analysis to the visibility of gratings. J Physiol (Lond) 1968; 197: 551-6.

24 Editorial. Spatial contrast sensitivity. Br J Ophthalmol 1978; 62: 197.

25 Arden GB. The importance of measuring contrast sensitivity in cases of visual disturbance. Br J Ophthalmol 1978; 62: 198-209.

26 Vacgan, Halliday BL. A forced-choice test improves clinical contrast sensitivity testing. Br J Ophthalmol 1982; 66: 477-91.

27 Arden GB. Visual loss in patients with normal visual acuity. Trans Ophthalmol Soc UK 1978; 98: 219-31.
28 Hirsch J, Puklin JE. Reduced contrast sensitivity may precede clinically observable retinopathy in type I diabetes. In: Henkind $\mathrm{P}$, ed. Acta XXIV International Congress of Ophthalmology, S Francisco, 1982. Philadelphia: Lippincott, 1983: 719-24.

29 National Diabetes Data Group. Classification and diagnosis of diabetes mellitus and other categories of glucose intolerance. Diabetes 1979; 28: 1039-57.

30 Kinnear PR, Aspinall PA, Lakowski R. The diabetic eye and colour vision. Trans Ophthalmol Soc UK 1972; 92: 69-78.

31 Shalka HW. Effect of age on Arden grating acuity. $\mathrm{Br} J \mathrm{Oph}$ thalmol 1980; 64: 21-3.

32 Vaegen. The clinical value of printed contrast sensitivity tests. $J$ Physiol (Lond) 1980; 300: 76P.

33 Sekuler R, Hutman IP, Owsley CJ. Human aging and spatial vision. Science 1980; 209: 1255-6.

34 Sekuler R, Hutman IP. Spatial vision and aging. Contrast sensitivity. J Gerontol 1980; 35: 692-9.

35 Beazley LD, Illingworth DJ, John A, Greer DV. Contrast sensitivity in children and adults. BrJ Ophthalmol 1980; 64: 863-6.

36 Hutman LP, Sckuler R. Spatial vision and aging. Criterion effects. J Gerontol 1980; 35: 700-6.

37 Arden GB. Visual loss in patients with normal visual acuity. Trans Ophthalmol Soc UK 1978; 98: 219-23. 